\title{
DNA biosensors and biomarkers to cancer detection
}

\begin{abstract}
Cancer is the leading cause of mortality in the worldwide, with around 8.8 million deaths in 2015 in accordance with World of Health organization (WHO). ${ }^{1}$ For this reason, this disease has a particular research interest in order to reduce the prevalence and mortality rates. Biomarkers in cancer provide a powerful approach to understand this complex disease as well as giving the possibility of timely diagnosis in a noninvasive way and it has different applications in epidemiology, diagnosis, progression and prognosis. At the other side, the Biosensors are instrument employing biological recognition properties for a selective bio-analysis and converts the molecular recognition signal to an electrical signal. Both technologies can support each one; biosensors can discover new biomarkers and biomarkers that drive the construction of new biosensors. This opinion will briefly summarize the "pros and cons" of this technologies to cancer detection, follow up and prognosis.
\end{abstract}

Volume 4 Issue I - 2018

\author{
Angela P Beltrán,',2 Mary García' \\ 'Pontificia Universidad Javeriana, Colombia \\ ${ }^{2}$ Colscience Institute, Colombia
}

\begin{abstract}
Correspondence: Angela Patricia Beltrán Lopez, Pontificia Universidad Javeriana, Human Genetic Institute, Carrera 7\# 4062, Bogotá, Colombia, Tel +57/3208320, Ext 2787, Email angelapbeltran@unal.edu.co
\end{abstract}

Received: February 09, 2018 | Published: February 15, 2018

Keywords: cancer, biomarkers, biosensors, detection

Abbreviations: mRNA, messenger RNA; MiRNA, micrornas; ctDNA, cell tumoral DNA; POC, point of care

\section{Introduction}

Cancer is one of the leading causes of death worldwide, and was responsible for 8.8 million deaths in 2015. Globally, nearly one in six deaths is due to cancer. ${ }^{1}$ Cancer is a genetic disease where genetics and epigenetics changes can occur, these changes guide to altered gene or protein expression or altered protein composition of cells resulting in alteration of the cycle and cell growth which translates in tumoral phenotypes. Environmental factors (such as diet, lifestyle and exposure to ultraviolet radiation or carcinogenic pollutants) play a major role in the development of some cancer. Genetic changes and environmental factors interact to influence tumor development. ${ }^{2}$ Biomarkers and biosensor take advantage of this characteristic of cancer cell to detect certain molecules like DNA, DNA modifications, RNA, proteins or protein modifications or other biological molecules produce by the tumor itself or by the cells around the tumor in response to the presence of cancer. Early detection in cancer is crucial to give an appropriate treatment and to get better outcomes in near and long time in the patients. Biomarkers and biosensors have shown different interesting results on the cancer detection, monitoring and therapy response highlighting the minimally invasive or non-invasive collection of samples. Thus, for example, biomarkers based on protein like CA 15-3 for detection of breast cancer and CA 125 for recurrence of ovarian cancer, or more recently biomarkers in reference to epigenetic changes of gene methylation in breast and ovarian cancer. ${ }^{3}$ In the case of carcinoma Hepatocellular, the epigenetic modifications of the gene promoter that involved the synthesis of cyclin-dependent kinase inhibitors $\mathrm{p} 15, \mathrm{p} 16$ and RASSF1A, represent potentially valuable biomarkers for the early and preclinical diagnosis. ${ }^{4}$ The last approximation cancer SEEK, which combine genetic markers and protein markers for detection up to five different types of cancer (ovary, liver, stomach, pancreas and esophagus), and estimate the origin of tumor cells in a significant proportion of cases. ${ }^{5}$ Additionally, have been studied miRNA, markers with better capacity to predict cancer than DNA or mRNA since a single molecule of miRNA has the capacity to regulate more than one hundred mRNA, with which they have orders of magnitude much greater in terms of the information about the prognosis that they provide us. Currently different molecular biology tools had permitted to develop molecular tumor signatures based on mRNA, miRNA and $\mathrm{DNA}^{6}$ that can help in tumor classification, subtype and ability to respond to certain therapies nowadays several of these are used in clinical practice. ${ }^{7}$

Biomarkers based on proteins (protein biomarkers), have certain disadvantages over genomic markers. In the studies carried out by Dawson and Bettegowda show that circulating DNA might perform better than the protein biomarkers; being ctDNA more sensitive than protein biomarkers when it comes to detecting breast ${ }^{8}$ and bowel cancer, ${ }^{9}$ respectively. Although protein biomarkers are used in the clinical practice, it can present false positives because there are other reasons that it can be elevated in the blood. Contrary false positives are rarer with ctDNA because it is defined by mutations and other genomic changes that are hallmarks of cancer cells specific to time and space. ${ }^{10}$ Other important disadvantage of biomarker in cancer detection is the use of single markers while in some cases is specifics; in others, it may not detect the variability of disease through the population, which lowers accuracy, sensitivity and specificity. Therefore, the results published should be validated in other patient samples, in order to detect the sensitivity of the detection and quantification based on body fluids studied.

The common techniques to detect biomarkers are based on enzyme-linked immunosorbent assay (ELISA), polymerase chain reaction (PCR) or even wasteful technique such as high performance liquid chromatography (HPLC) specific of histone change or bisulfite modification sequencing. These have technological limitations like slow detection and expensive reagents in every trial. ${ }^{11}$ For this reason, biosensors play an important role in biomarker detection. Biosensors are designed to detect a specific biological analyte by essentially converting a biological molecule (ie, protein, DNA, RNA) into an electrical signal that can be detected and analyzed. ${ }^{12}$ 
The recently advances in biosensors to cancer detection include biosensors with electrochemical detection, optical and mass-based detection techniques. ${ }^{13}$ Biosensor technology has received heightened interest over the past decade, since it is a promising candidate for lower detection limit with rapid analysis time at relatively low cost. Technological advances have provided different tools and materials needed to construct biosensors, which integrated with microfluidic system, probe, sampler, detector, amplifier and logic circuitry. For medical application, this cost advantage will allow the development of extremely low cost, disposable biochips that can be used for in-home medical diagnostics of diseases without the need of sending samples to a laboratory for analysis which time consuming. ${ }^{14}$ Unfortunately, the implementation of this biosensors into the clinical routine practice have not been successful, probably for the high degree of complexity of the cancer cell where a single biomarker may be involved in multiple cellular processes and because the biosensors specific to detect molecular markers are not reproducible in cancer approach. ${ }^{15}$

\section{Conclusion}

Biomarkers have gained greater recognition and application in clinical practice, mainly used for detecting and monitoring cancer, however, until now, biomarkers are not completely reliable, because in some cases they can be altered by different medical OR population conditions. Molecular profiling generates a vast amount of highly complex data, with millions of potential biomarkers, but a rigorous assay and validation is necessary to obtain biomarkers with high selectivity and specificity, also, some biomarkers have been tested in certain population groups and the genetic variability can affect the use of these markers in different population groups. Biosensors Genomic and proteomic profiles, as well as next generation sequencing, can aid in the discovery of new specific biomarkers for biosensors use, and it is important to consider certain characteristics, as it should be specific and respond only just to a disease state. Although some efforts have been made to design biosensors for detecting multiple biomarkers simultaneously, ${ }^{16}$ these studies expand the use clinical profile as cancer and the impact of early detection. Another challenge is the miniaturization and integration of microfluidic technologies to obtain a better detection of multiplex and devices with integrated functions. The uses of biosensors in the early detection, monitoring and state of cancer are hopeful keys to effective treatment, which will reduce the mortality rate of patients.

\section{Acknowledgements}

None.

\section{Conflict of interest}

The authors report no conflicts of interest in this work.

\section{References}

1. Ferlay J, Ervik M, Dikshit R, et al. Cancer Incidence and Mortality Worldwide: sources, methods and major patterns in GLOBOCAN 2012. Int J Cancer. 2012;136(5):E359-386.

2. Rasooly A, Jacobson J. Development of biosensors for cancer clinical testing. Biosens Bioelectron. 2006;21(10):1851-1858.

3. Ahluwalia A, Yan P, Hurteau JA, et al. DNA methylation and ovarian cancer. I. Analysis of $\mathrm{CpG}$ island hypermethylation in human ovarian cancer using differential methylation hybridization. Gynecol Oncol. 2001;82(2):261-268

4. Rivenbark AG, Coleman WB. The use of epigenetic biomarkers for preclinical detection of hepatocellular carcinoma: potential for noninvasive screening of high-risk populations. Clinical Cancer Research. 2007;13(8):2309-2312.

5. Cohen JD, Li L, Wang Y, et al. Detection and localization of surgically resectable cancers with a multi-analyte blood test. Science. 2018;359(6378):926-930.

6. Mamatjan Y, Agnihotri S, Goldenberg A, et al. Molecular signatures for tumor classification: an analysis of the cancer genome atlas data. $\mathrm{J} \mathrm{Mol}$ Diagn. 2017;19(6):881-891.

7. Lal S, Mc Cart Reed AE, De Luca XM, et al. Molecular signatures in breast cancer. Methods. 2017;131:135-146.

8. Dawson SJ, Tsui DWY, Murtaza M. Analysis of circulating tumor DNA to monitor metastatic breast cancer. N Engl J Med. 2013;368(13):11991209.

9. Bettegowda C, Sausen M, Leary RJ, et al. Detection of circulating tumor DNA in early- and late-stage human malignancies. Sci Transl Med. 2014;6(224):224ra24.

10. Yong E. Cancer biomarkers: Written in blood. Nature. 2014;511(7511):524-526.

11. Rusling JF, Challa V Kumar, J Silvio Gutkind, et al. Measurement of biomarker proteins for point-of-care early detection and monitoring of cancer. Analyst. 2010;135(10):2496-2511.

12. Bohunicky B, Mousa SA. Biosensors: the new wave in cancer diagnosis. Nanotechnol Sci Appl. 2011;4:1-10.

13. Vigneshvar S, Sudhakumari CC, Senthilkumaran B, et al. Recent Advances in Biosensor Technology for Potential Applications - An Overview. Frontiers in Bioengineering and Biotechnology. 2016;4:11.

14. Perumal V, Hashim U. Advances in biosensors: Principle, architecture and applications. Journal of Applied Biomedicine. 2014;12(1):1-15.

15. Villarreal-Gómez L, et al. Detection of molecular markers of cancer through the use of biosensors. Biology and Medicine. 2015;S2:5.

16. Parra-Cabrera C, Samitier J, Homs-Corbera A. Multiple biomarkers biosensor with just-in-time functionalization: Application to prostate cancer detection. Biosensors and Bioelectronics. 2016;77:1192-1200. 